\title{
Lehrstuhlportrait: Lehrstuhl für Entrepreneurship am Institut für Technologiemanagement an der Universität St. Gallen
}

\section{Dietmar Grichnik}

(1) Der Lehrstuhl für Entrepreneurship kurz und knapp:

- Prof. Dr. Dietmar Grichnik ist Ordinarius für Entrepreneurship und Direktor des Instituts für Technologiemanagement.

- Mitarbeitende: ein Projektleiter, drei wissenschaftliche Mitarbeiter, eine persönliche Assistentin, zwei Research Affiliates, zwei studentische Mitarbeiter.

- Prof. Dietmar Grichnik ist u. a. Mitglied im Präsidium des FGF - Förderkreis Gründungsforschung e. V., Mitgründer und Coach verschiedener Start-ups aus dem Hochschulbereich sowie Editor, Reviewer und Programmkomiteemitglied bei diversen Journalen und Konferenzen. Seine Aktivitäten werden in führenden Fachzeitschriften wie dem Journal of Business Venturing, Entrepreneurship Theory \& Practice, Journal of Economic Behavior \& Organization, Die Betriebswirtschaft, Zeitschrift für Betriebswirtschaft und Zeitschrift für betriebswirtschaftliche Forschung veröffentlicht.

\section{(2) Grundausrichtung des Lehrstuhls}

Forschung und Lehre an unserem Lehrstuhl sind dem unternehmerischen Handeln und Denken gewidmet. Wir bieten dabei eine internationale Plattform für den wissenschaftlichen und praxisbezogenen Austausch zu den verschiedenen Themenbereichen des Entrepreneurships in neugegründeten wie auch bereits etablierten Unternehmen. Aus einer entscheidungsorientierten Perspektive untersuchen wir Fragestellungen zum unternehmerischen Prozess der Entdeckung, Schaffung, Bewertung und Ausschöpfung unternehmerischer Gelegenheiten, die eine hohe Relevanz für die internationale Forschung und die unternehmerische Praxis haben. Unser interaktiv ausgerichtetes Lehrkonzept zielt auf die Vermittlung fundierter theoretischer Kenntnisse und praxisorientierter Fähigkeiten für unternehmerisches Denken und Handeln.

Darüber hinaus entwickeln und vermitteln wir innovative Konzepte, Methoden und Instrumente, mit denen wir Entrepreneure an der Universität St. Gallen bei ihren unternehmerischen Entscheidungen ermutigen und unterstützen wollen. Inhaltlich widmet sich das Lehrstuhlteam fünf Schwerpunktbereichen. Die nachfolgende Abbildung führt die aktuellen Arbeitsbereiche des Lehrstuhls auf. 


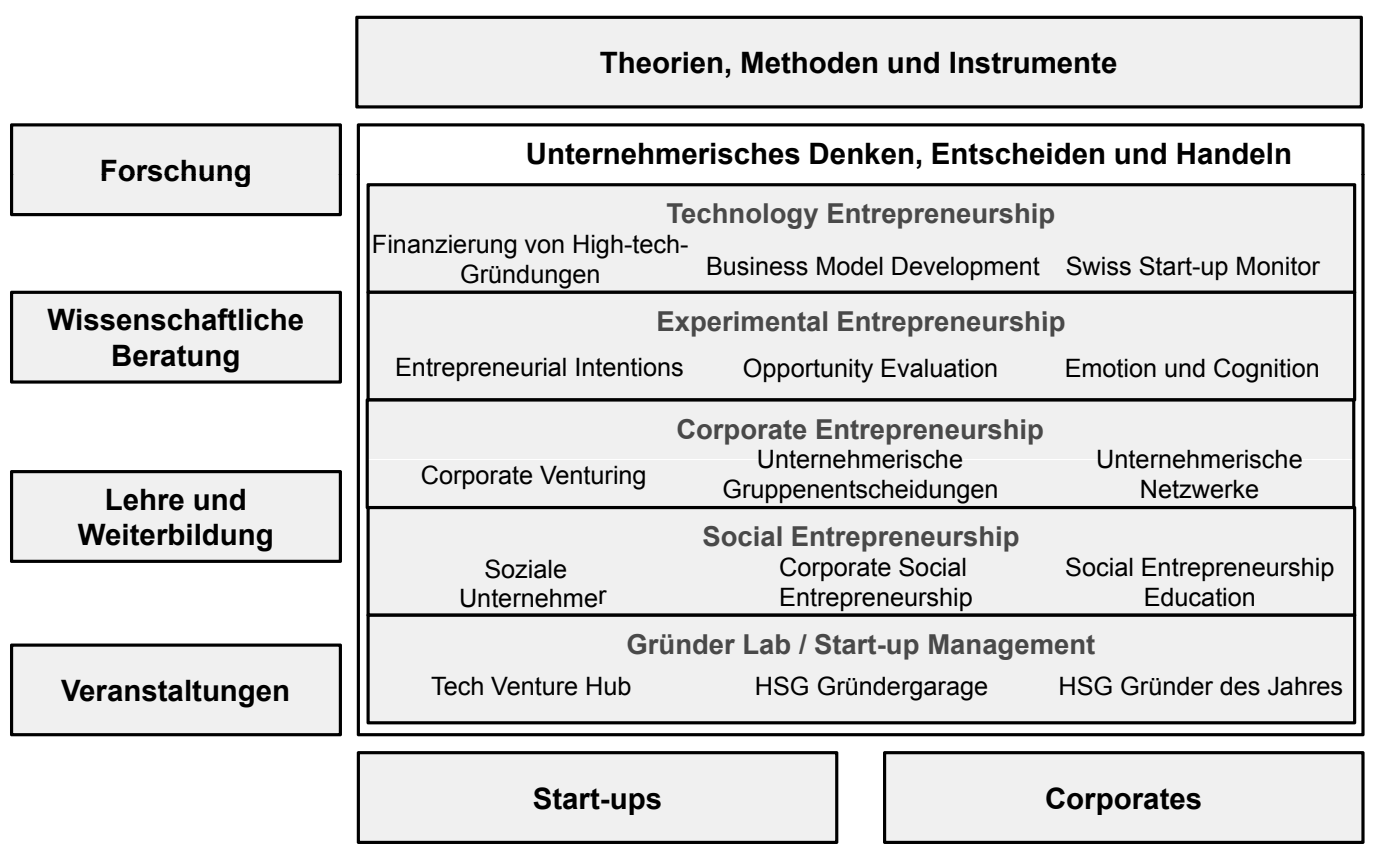

\section{(3) Team}

Seit August 2010 leitet Prof. Dr. Dietmar Grichnik den Lehrstuhl für Entrepreneurship am Institut für Technologiemanagement der Universität St. Gallen. Zuvor hatte Prof. Grichnik von 2007 bis 2010 den Lehrstuhl für Unternehmertum und Existenzgründung an der WHU - Otto Beisheim School of Management in Vallendar sowie den Lehrstuhl für Corporate Finance \& Entrepreneurship an der Universität Witten/Herdecke (2005 - 2007) inne. Nach dem Studium der Betriebswirtschaftslehre und der Wirtschaftspädagogik an der Universität zu Köln, wurde er im Jahr 2000 an der Universität zu Köln promoviert. 2005 habilitierte er sich an der Heinrich-Heine-Universität Düsseldorf.

$\mathrm{Zu}$ seinen Forschungs- und Lehrgebieten zählen insbesondere das Entscheidungs- und Risikoverhalten von Unternehmern im internationalen Kontext sowie die Gründungs- und Wachstumsfinanzierung (Entrepreneurial Finance).

Neben Prof. Grichnik sind Dr. Christian Koropp (Projektleiter Competence Center Technology Entrepreneurship), die wissenschaftlichen Mitarbeitenden Maria Fantetti, Ronny Baierl und Florian Forster sowie die Research Affiliates Dr. Rainer Lauterbach und Katrin Haug am Lehrstuhl tätig. Administrativ wird das Lehrstuhlteam von Elke Feyerherd als persönliche Assistentin von Prof. Grichnik unterstützt. 


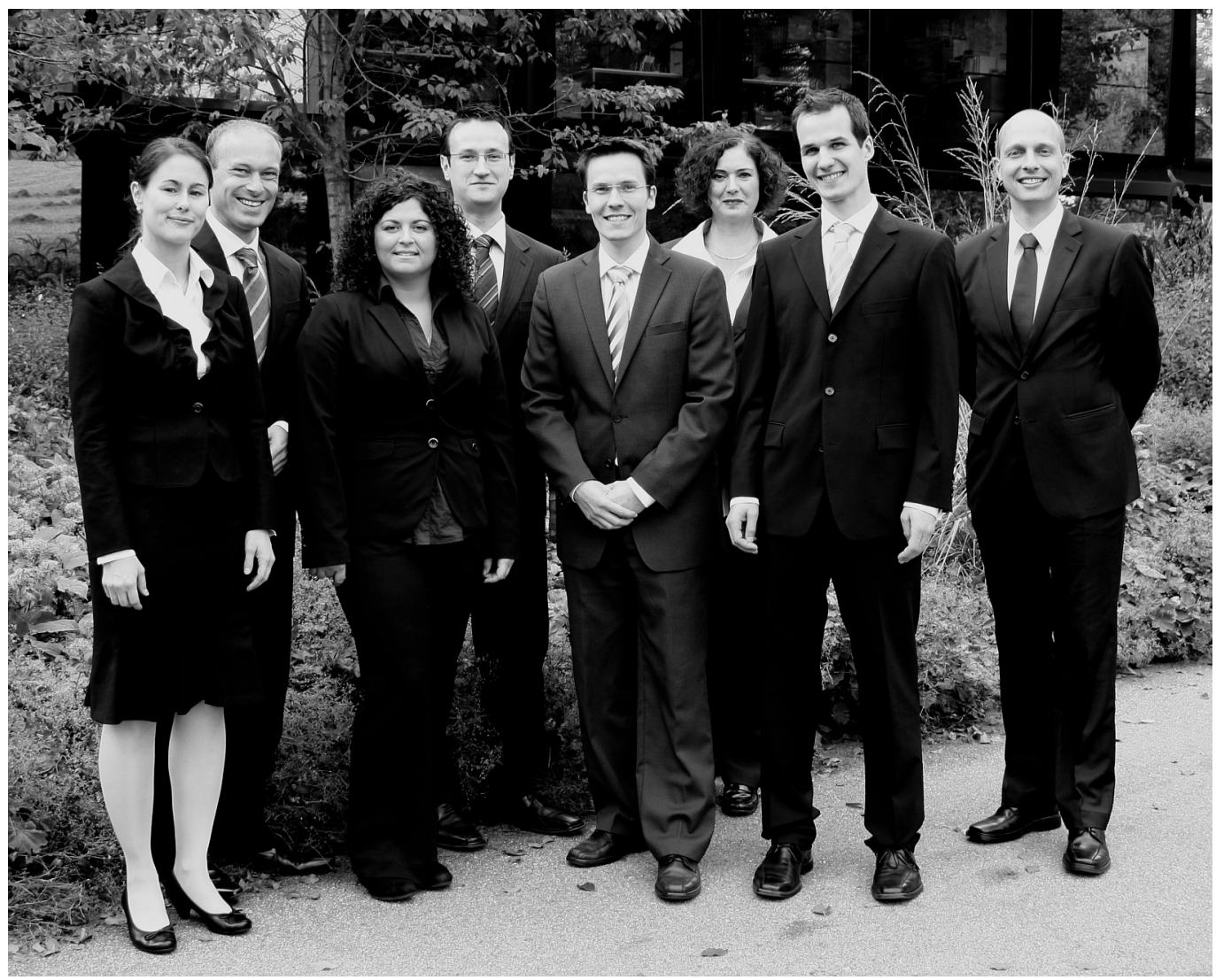

\section{(4) Forschung}

Das wesentliche Ziel unseres Lehrstuhls ist, einen substantiellen Beitrag zum wissenschaftlichen Fortschritt in der Entrepreneurshipforschung zu leisten und die Schweiz als Wissenschafts- und Innovationsstandort weiter zu stärken. Zu diesem Zweck führen wir fundierte theoretische und empirische Projekte mit Implikationen für Forschung, Lehre und Praxis durch. Verwirklicht werden die Projekte in vielfältigen Kooperationen mit führenden nationalen und internationalen Partnern. Exemplarisch für aktuelle Projektinitiativen seien hier der „Swiss Start-up Monitor“ und das Forschungsprojekt „Financing High-tech Entrepreneurship“ erwähnt.

Das Projekt "Swiss Start-up Monitor" wird in Zusammenarbeit mit der ETH Zürich und der Universität Basel durchgeführt. Mit diesem Monitor soll die schweizweit einzige, unabhängige Datenbank zum sicheren Erfassen, Verwalten und Austauschen von quantitativen Start-up-Performance-Daten errichtet werden. Der Initiative liegt ein innovatives, web-basiertes Konzept zugrunde, bei dem Start-ups erstmalig ihre Daten selber erfassen können und völlige Transparenz über die Verwendung ihrer Daten haben. Im Laufe der Zeit werden die dadurch erlangten Daten über Jahre hinweg verglichen werden können und bieten somit eine ideale Basis für wissenschaftliche und praxisrelevante Auswertungen. Hierdurch soll die Entwicklung von potenzialreichen Start-ups in der Schweiz effizient nach Regionen, Industrien und Universitäten verfolgt und der wirtschaftliche Nutzen 
von Unternehmensgründungen in der Schweiz aufgezeigt werden können. Das Projekt wird von der Kommission für Technologie und Innovation (KTI), der Gebert Rüf Stiftung und der AVINA Stiftung finanziell unterstützt.

Das vom Grundlagenforschungsfonds (GFF) der Universität St. Gallen geförderte Forschungsprojekt „Financing High-tech Entrepreneurship“ wird von unserem Lehrstuhl zusammen mit dem Zentrum für Europäische Wirtschaftsforschung (ZEW) in Mannheim durchgeführt. High-tech Start-ups sind ein wichtiger Eckpfeiler der volkswirtschaftlichen Entwicklung; ihre Überlebensrate ist im Vergleich $\mathrm{zu}$ weniger technologieorientierten Start-ups allerdings ungemein kleiner, welches nicht zuletzt an ihrer finanziellen Ausstattung liegt. Hier setzt das Forschungsprojekt an und analysiert die Finanzierungsstrategien deutscher High-tech Start-ups und deren Erfolgsauswirkungen anhand der Daten des KfW/ZEW Gründungspanels.

\section{(5) Lehre}

Das Lehrkonzept des Lehrstuhls für Entrepreneurship ist auf „Excellence in Entrepreneurship Education“ ausgerichtet. Es zielt darauf ab, den Studenten der Bachelor-, Masterund Doktoratsstufe an der Universität St. Gallen ein theoretisch fundiertes Wissen und zugleich anwendbare Best Practice zu den verschiedenen Facetten des unternehmerischen Entscheidens und Handelns in neugegründeten und etablierten Unternehmen zu vermitteln. Im Bachelorprogramm und im IMT-Masterprogramm werden daher jeweils grundlagentheoretische Veranstaltungen, z. B. zum Thema Technologie und Entrepreneurship, wie auch praxisorientierte Projektseminare, in denen zum Beispiel die Ausarbeitung eines Geschäftsmodells oder eines Businessplans in interdisziplinären Teams vermittelt wird, angeboten. In den Kursen der Doktoratsstufe werden vor allem aktuelle Themen der Entrepreneurshipforschung diskutiert und die Anschluss- und Publikationsfähigkeit der eigenen Forschungsarbeiten der Doktorierenden in der internationalen Forschung gefördert.

Die Inhalte unseres Lehrprogramms stützen sich auf aktuelle Erkenntnisse aus den Forschungsarbeiten des Lehrstuhls und anderer renommierter Fachvertreter. In Anlehnung an die angelsächsische Kursdidaktik werden in unseren Lehrveranstaltungen moderne und interaktive Lehrmethoden, z. B. Fallstudienarbeit, Expertenvorträge und Praxisprojekte eingesetzt.

\section{(6) Gründungsförderung}

Die vielfältigen Aktivitäten des Lehrstuhls sind Bestandteil des Schwerpunktes Business Innovation an der HSG. Neben Forschung und Lehre ist es unserem Lehrstuhl ein besonderes Anliegen, die Gründungskultur an unserer Universität aktiv zu fördern. Mit Unterstützung des Rektorats der Universität St. Gallen und der HSG Alumni wurde hierfür der HSG Entrepreneurship Campus ins Leben gerufen. Ein wesentliches Element hierbei ist das von Florian Forster geleitete HSG Gründerlab, welches Studierenden aber auch Alumni, Wissenschaftlern und Mitarbeitern auf dem Campus der Universität eine unabhängige Anlaufstelle für innovative und technologieorientierte Geschäftsideen bietet. Gezielt werden dabei auch Alumni unserer Universität in die Förderung und das Mentoring einbezogen. Die Leistungen des HSG Gründerlab für Gründungsinteressierte umfassen:

- Spezifische Gründungsberatung

- Entwicklung des Geschäftsmodells 
- Erstellung von Businessplänen

- Interdisziplinäre Teambildung

- Zugang zu Gründer- und Förderprogrammen

- Kontakt zu passenden Investoren

- Breites Netzwerk im In- und Ausland

Einmal jährlich veranstaltet der Lehrstuhl zudem die HSG Gründergarage, mit der die Sichtbarkeit der Gründungskultur an der Universität St. Gallen gestärkt und Universitätsangehörige für das Thema Entrepreneurship sensibilisiert werden sollen. Die Gründergarage findet jeweils zu Beginn des Frühjahrssemester statt. Mehr als 50 Aussteller, bestehend aus Studierenden, Wissenschaftlern, Mitarbeitern und Alumni der HSG, stellen sich von morgens bis spät in den Abend in der Garage vor und diskutieren lebhaft mit Gründungsinteressierten unserer Hochschule. Kontinuierliche Informationen zur Gründerszene an der Universität finden sich zudem auf dem HSG Start-up-Blog (www.startup.unisg.ch), der im Dezember 2010 gestartet wurde.

Im Rahmen der Gründergarage wird ferner der mit 10'000 CHF dotierte Wettbewerb „HSG Gründer des Jahres“ durchgeführt. Im Rahmen des Wettbewerbs werden innovative und forschungsbasierte Gründungen von Universitätsangehörigen durch eine Fachjury prämiert. Ziel des Wettbewerbs ist, zu herausragenden Unternehmensgründungen aus der Universität anzuregen und diese sichtbar zu machen. Zur Gründergarage wurden auch das HSG Alumni Start-up Mentoring-Programm und der HSG Alumni Idea-Check aufgelegt. Beide Projekte bringen unternehmerische Studierende mit branchenerfahrenen Alumni zusammen, um Geschäftsideen von Universitätsangehörigen zu bewerten und bis zur Marktreife $\mathrm{zu}$ begleiten.

\section{(7) Ausgewählte Publikationen}

\section{Bücher}

Grichnik, D./Witt, P. (Hrsg.) (2011): Entrepreneurial Marketing, ZfB-Ergänzungsheft, Wiesbaden. Grichnik, D./Brettel, M./Koropp, C./Mauer, R. (2010): Entrepreneurship. Unternehmerisches Denken, Entscheiden und Handeln in innovativen und technologieorientieren Unternehmungen, Stuttgart.

Grichnik, D. (2006): International Entrepreneurship: Entscheidungs- und Risikoverhalten von Unternehmensgründern und Venture-Finanziers in kulturellen Kontexten - Theoriebildung und empirische Analysen, Reihe Entrepreneurship, Marketing \& Innovation, Berlin.

Börner, C.J./Grichnik, D. (Hrsg.)(2005): Entrepreneurial Finance - Kompendium der Gründungsund Wachstumsfinanzierung, Berlin, Heidelberg.

\section{Artikel in wissenschaftlichen Journalen}

Mauer, R./Grichnik, D. (2011): Dein Markt, das unbekannte Wesen : Zum Umgang mit Marktunsicherheit als Kern des Entrepreneurial Marketing, in: Zeitschrift für Betriebswirtschaft, Special Issue $6 / 2011$.

Welpe, I./ Spörrle, M./ Grichnik, D./Michl, T./ Audretsch, D.(2011): Emotions and Opportunities: The Interplay of Opportunity Evaluation, Fear, Joy, and Anger as Antecedent of Entrepreneurial Exploitation, in: Entrepreneurship Theory and Practice, Jg. 35, Nr. 9. 
Faschingbauer, M./Grichnik, D. (2011): Effectuation : Das Unternehmerische im Unternehmen wecken. In: Zeitschrift Führung + Organisation. Jg. 80, Nr. 5, S. 337-344.

Grichnik, D./ Smeja, A./ Welpe, I.(2010): The Importance of Being Emotional: How do Emotions Affect Entrepreneurial Opportunity Evaluation and Exploitation?, in: Journal of Economic Behavior and Organization, Jg. 76, Nr. 1, S. 15-29.

Grichnik, D./ Singh, L. (2010): Resource Bootstrapping of Nacent Entrepreneurs: Conscious Entrepreneurial Decision or Forced Reaction?, in: Frontiers of Entrepreneurship Research, Nr. 1, S. 500-514.

Brinckmann, J./Grichnik, D./Kapsa, D. (2010): Should entrepreneurs plan or just storm the castle? A meta-analysis on contextual factors impacting the business planning - performance relationship in small firms, in: Journal of Business Venturing Jg. 25, Nr. 1, S. 24-40.

Börner, C. J./Grichnik, D./Reize, F. (2010): Finanzierungsentscheidungen mittelständischer Unternehmer - Einflussfaktoren der Fremdfinanzierung deutscher KMU. In: Zeitschrift für betriebswirtschaftliche Forschung Jg. 62, Nr. 3, S. 227-250.

\section{Buchkapitel}

Grichnik, D./Koropp, C. (2011): Business Angels and Energy Investing: Insights from a German Panel Study, in: Wüstenhagen, R./Wuebker, R. (Hrsg.): Handbook of Research on Energy Entrepreneurship, Cheltham, S. 197-213.

Grichnik, D. (2009): Policy Issues in Financing Entrepreneurship, in: OECD Report Strengthening Entrepreneurship and Economic Development in East Germany: Lessons from Local Approaches, S. 109-132.

Koropp, C./Grichnik, D. (2009): Sedo.com : The Founder's Dilemma: When Is the Right Time for Exiting?, in: Hisrich, R.D. (Hrsg.): International Entrepreneurship: Starting, Developing, and Managing a Global Venture. London, S. 416-426.

Harms, R./Grichnik, D. (2008): Entrepreneurship als eigenständige Disziplin -Trendwende im Selbstverständnis der deutschsprachigen Forschungsszene?, in: Stand und Perspektiven der deutschsprachigen Entrepreneurship- und KMU-Forschung. Stuttgart, S. 29-47.

Grichnik, D./von Kortzfleisch, H./Magin, P. (2008): Open Scientific Entrepreneurship Engineering Ein offener, ganzheitlicher und systematischer Ansatz zur Unterstützung von Existenzgründern aus Hochschulen, in: Academic Entrepreneurship: Unternehmertum in der Forschung, S. 1-23.

Anschrift: Universität St. Gallen, Institut für Technologiemanagement, Lehrstuhl für Entrepreneurship, Dufourstrasse 40 a, CH-9000 St. Gallen, Tel.: +41(0)71/224-72-00, Fax: +41(0)71/224-73-01, Email: dietmar.grichnik@unisg.ch, URL: www.item.unisg.chlent 